\title{
The FIREBall fiber-fed UV spectrograph
}

\author{
Sarah E. Tuttle ${ }^{a}$, David Schiminovich ${ }^{a}$, Bruno Milliard ${ }^{b}$ Robert Grange $^{b}$, D. Christopher Martin ${ }^{c}$, \\ Shahinur Rahman ${ }^{c}$, Jean-Michel Deharveng ${ }^{b}$, Ryan McLean ${ }^{c}$, Gordon Tajiri ${ }^{a}$, M. Matuszewski ${ }^{c}$ \\ ${ }^{a}$ Columbia University, Pupin Hall, 550 W. $120^{\text {th }}$ St., New York, NY 10032, United States \\ ${ }^{b}$ Observatoire d'Astronomie Marseille Provence, Pole Etoile Site Chateau Gombert, 38, Rue \\ Frederic Joliot-Curie, 13388 Marseille cedex 13, France \\ ${ }^{c}$ California Institute of Technology, MC 405-47, 1200 East California Boulevard, Pasadena, CA \\ 91125, United States
}

\begin{abstract}
FIREBall (Faint Intergalactic Redshifted Emission Balloon) had a successful first engineering flight in July of 2007 from Palestine, Texas. Here we detail the design and construction of the spectrograph. FIREBall consists of a $1 \mathrm{~m}$ telescope coupled to a fiber-fed ultraviolet spectrograph flown on a short duration balloon. The spectrograph is designed to map hydrogen and metal line emission from the intergalactic medium at several redshifts below $z=1$, exploiting a small window in atmospheric oxygen absorption at balloon altitudes. The instrument is a wide-field IFU fed by almost 400 fibers. The Offner mount spectrograph is designed to be sensitive in the $195-215 \mathrm{~nm}$ window accessible at our altitudes of $35-40 \mathrm{~km}$. We are able to observe Lya, as well as OVI and CIV doublets, from $0.3<\mathrm{z}<0.9$. Observations of UV bright B stars and background measurements allow characterization of throughput for the entire system and will inform future flights.
\end{abstract}

Keywords: UV, fiber, IFU, spectroscopy, astronomical instrumentation, galaxy evolution, balloon-borne instrument

\section{INTRODUCTION}

The warm-hot component of the IGM (warm hot intergalactic medium - WHIM), predicted to have temperatures between $10^{5}-10^{7} \mathrm{~K}$, is heated both by collapse during structure formation and from energy injected by galactic winds ${ }^{1}$. Gas at these temperatures emits lines in the ultraviolet arising from recombination and collisional excitation, with the stronger emission occurring in regions of highest density nearest to galaxies and clusters. Our experiment uses a small window in $\mathrm{O}_{2}$ and $\mathrm{O}_{3}$ atmospheric absorption to search for these emission signatures ${ }^{2}$. The experiment bandpass is 197$213 \mathrm{~nm}$, allowing observations of CIV $(\lambda \lambda 1548,1551)$, Ly $\alpha(1216)$ and OVI $(\lambda \lambda 1032,1038)$ at redshifts of $0.3,0.6$ and 0.9 , respectively. This method complements the current approach of QSO line-of-sight absorption line mapping by targeting the three dimensional distribution of matter rather than along a pencil beam. The FIREBall spectrograph has a $2.5^{\prime} \times 2.5^{\prime}$ field of view, well-matched to the expected diffuse nature of the IGM ${ }^{3}$.

\section{INSTRUMENT DESIGN AND ASSEMBLY}

\subsection{Experiment overview}

FIREBall is a $1 \mathrm{~m}$ balloon-borne telescope combined with a fiber-fed UV spectrograph ${ }^{4}$. The project is a collaboration between Caltech, LAM (Laboratoire d'Astrophysique de Marseille), CNES, and Columbia University. The initial experiment is configured for short duration flights. The stationary primary mirror is mounted above a $1.2 \mathrm{~m}$ siderostat that gives high precision guiding ( 3 " rms) through a combination of active and passive controls. This level of stability is necessary to provide the extended exposures $(>20,000 \mathrm{sec})$ on a given field needed to achieve the depths required for detections. The telescope is mounted for flight in a carbon-fiber gondola. This design gave the necessary stiffness for optical stability as well as the strength to withstand the $10 \mathrm{~g}$ landing forces. The telescope is designed to have $\mathrm{F} / 2.5$ to match the numerical aperture of the fibers (Table 1).

Ground-based and Airborne Instrumentation for Astronomy II, edited by lan S. McLean, Mark M. Casali, Proc. of SPIE Vol. 7014, 70141T, (2008) · 0277-786X/08/\$18 · doi: 10.1117/12.789836 
Table 1. Instrument Specifications

\begin{tabular}{|c|c|c|}
\hline Parameter & Value & Comments \\
\hline Telescope F/\# & 2.5 & \\
\hline Telescope Primary Diameter & $1.0 \mathrm{~m}$ & Siderostat (secondary) Diameter $=1.2 \mathrm{~m}$ \\
\hline Spectrograph F/\# & 2.5 & Offner style mount \\
\hline Spectrograph Resolution (R) & 5000 & \\
\hline Grating Groove Density & $5000 \mathrm{~g} / \mathrm{mm}$ & $4800 \mathrm{~g} / \mathrm{mm}$ for next flight \\
\hline $\begin{array}{l}\text { Fiber Numerical Aperture } \\
\text { (Polymicro FVP100110125) }\end{array}$ & .22 & $\begin{array}{l}\text { Core diameter }=100 \mu \mathrm{m} \\
\text { Total diameter }=125 \mu \mathrm{m}\end{array}$ \\
\hline Plate Scale & $12 \mu \mathrm{m} / \operatorname{arcsec}$ & \\
\hline Angular Resolution & $10 \operatorname{arcsec}$ & \\
\hline Field of View (total \& single fiber) & $\begin{array}{c}160 \times 160 \operatorname{arcsec}^{2} \\
8 \operatorname{arcsec}\end{array}$ & \\
\hline Total system efficiency & .005 & $\begin{array}{l}\text { Atmospheric transmission }=0.70 \\
\text { Primary efficiency }=0.90 \\
\text { Secondary efficiency }=0.90 \\
\text { Dichroic efficiency }=0.93 \\
\text { Fiber efficiency }=0.70 \\
\text { Offner efficiency }=0.90 \\
\text { Grating efficiency }=0.25 \\
\text { Detector efficiency }=0.06\end{array}$ \\
\hline Predicted signal / Sky background & $1900 \mathrm{LU} / 1000 \mathrm{LU}$ & \\
\hline
\end{tabular}

\subsection{In-flight requirements and tolerances}

The spectrograph was designed to handle the physical conditions that could be encountered during a balloon flight, requiring survival between $-20^{\circ}$ and $22^{\circ} \mathrm{C}$. The air temperature and spectrograph surface temperature was monitored during the flight. The internal temperature exceeded specifications during its time in the field awaiting retrieval. The operating temperature was designed to be $20^{\circ}+/-5^{\circ} \mathrm{C}$. This was achieved once float altitude was reached. We used a Minco patch heater system to ensure that the low temperatures outside the gondola did not affect detector performance. Both the spectrograph and the gondola itself were enclosed in insulating wraps. The spectrograph wrap was a standard MLI blanket.

Any acceptable design required that the detector not be at the atmospheric pressures found at $40 \mathrm{~km}$, roughly $1 / 1000^{\text {th }}$ atm. Field emission increases the frequency of dielectric breakdown and arcs. It was decided to enclose the entire spectrograph to allow a nitrogen environment and a pressure anywhere between full vacuum and $1 \mathrm{~atm}$. The enclosure was tested by the vendor both at full vacuum for 2 hours and overpressured to $1.1 \mathrm{~atm}$. The deflection of the top and base plate met the requirement of $<0.050$ ". The spectrograph was flown at $0.5 \mathrm{~atm}$ to allow for some conduction of heat while meeting the detector pressure requirements and minimizing deflection.

The mechanical design took into account the following optical constraints. The optical alignment specified positioning $+/-0.01 \mathrm{~mm}$ in the distance between optics. The tilt was required to be $+/-0.011 \mathrm{deg}$. A decenter of $+/-50 \mu \mathrm{m}$ was allowed. This led to the implementation of a two level hexapod structure, as well as a three-point mount for the grating. 


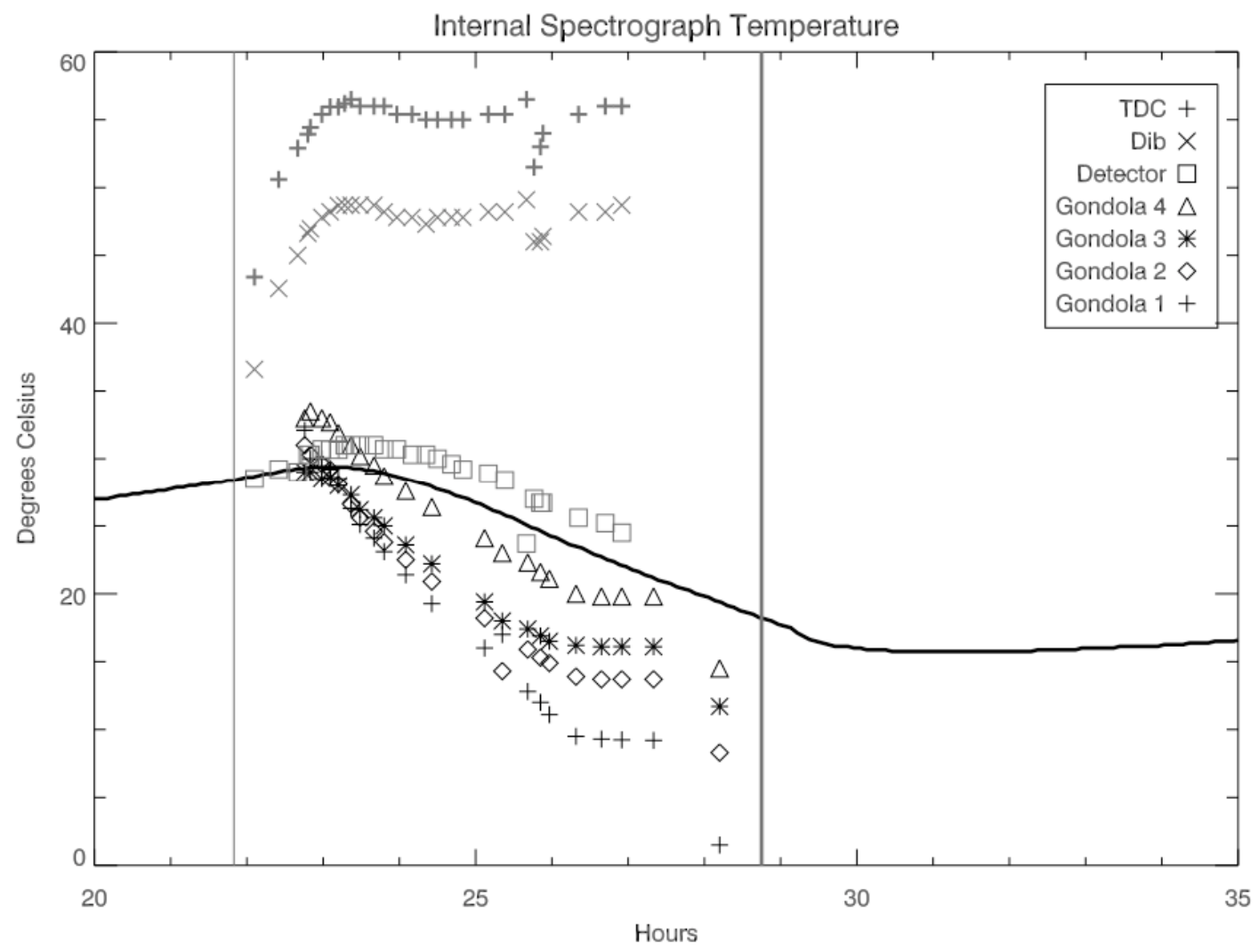

Figure 1: Internal temperature (bold horizontal line) from the flight. Launch is the first vertical line ( 22 hours) and landing is the second bold vertical line at $28.75 \mathrm{hrs}$. The internal temperature was recorded every five minutes. The TDC \& Dib temperatures are from the Front End Electronics for the spectrograph (housed in a separate can).

\subsection{Spectrograph design}

The objects being targeted by FIREBall are faint (1900 LU expected signal / 1000 LU background) and diffuse (100kpc physical scale). An all-reflective system eliminates chromatic aberrations as well as absorption introduced by lens materials at UV wavelengths. A wide-field NUV microchannel plate (MCP) detector (a Galaxy Evolution Explorer (GALEX) flight spare) was used. The optical design has been made compact for balloon flight by shifting the traditional Offner relay. This reduces the number of optics in the system. A fiber bundle was selected to feed the slit by virtue of its flexibility, transmission, and the availability of fibers to match object size. The convex grating is holographically ruled, decreasing the scattered light compared to a mechanically ruled grating. The entire spectrograph was placed in a vacuum enclosure to control pressure and isolate the optics from scattering light as well as from mechanical interference.

The double hexapod mount design was used for maximum adjustability during alignment and stiffness and stability during flight. The carbon fiber legs were lightweight and limited flexure. A locking sleeve was integrated with ball joint ends on each hexapod leg to secure the legs at the precise length needed for optical alignment (figure 2). Precision adjustments were made in leg length using a novel screw mechanism. The length was adjustable by twisting the carbon rod while holding one or both ball joints stationary. Each rod has one end with $1 / 4 "-20$ thread and the other end with a $1 / 4 "-28$ thread. If both ends are unlocked, this gives the ability to adjust the length 0.015 " per turn. In our estimations we could easily discern $1 / 4$ turns, giving the ability to control the length in steps of 0.0036 ". The threaded ends used for adjustments were epoxied into the carbon rods using AngstromBond 9119. Angled mount blocks (figure 2) were designed to reduce flexure in the system by approximating an ideal hexapod (three mount points per plate, rather than six). 

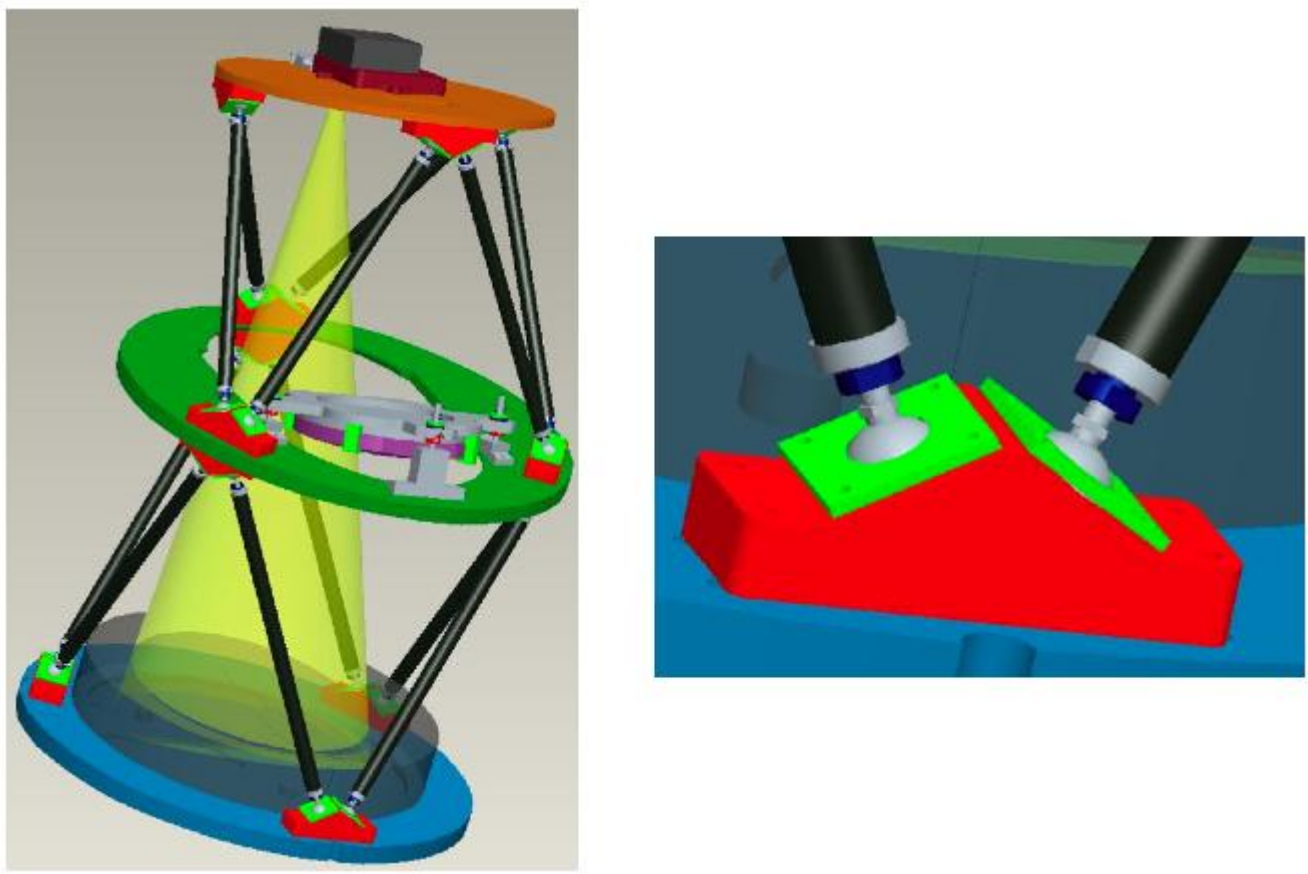

Figure 2: The internal double hexapod mount and mount plates (left) with incident light from the slit illuminating the Offner sphere (bottom). The left shows a zoom on the ball joints that mount each leg. The blue rings are the locking mechanism for the leg length. The green plates operate as a clamping spring to lock the location of each leg.
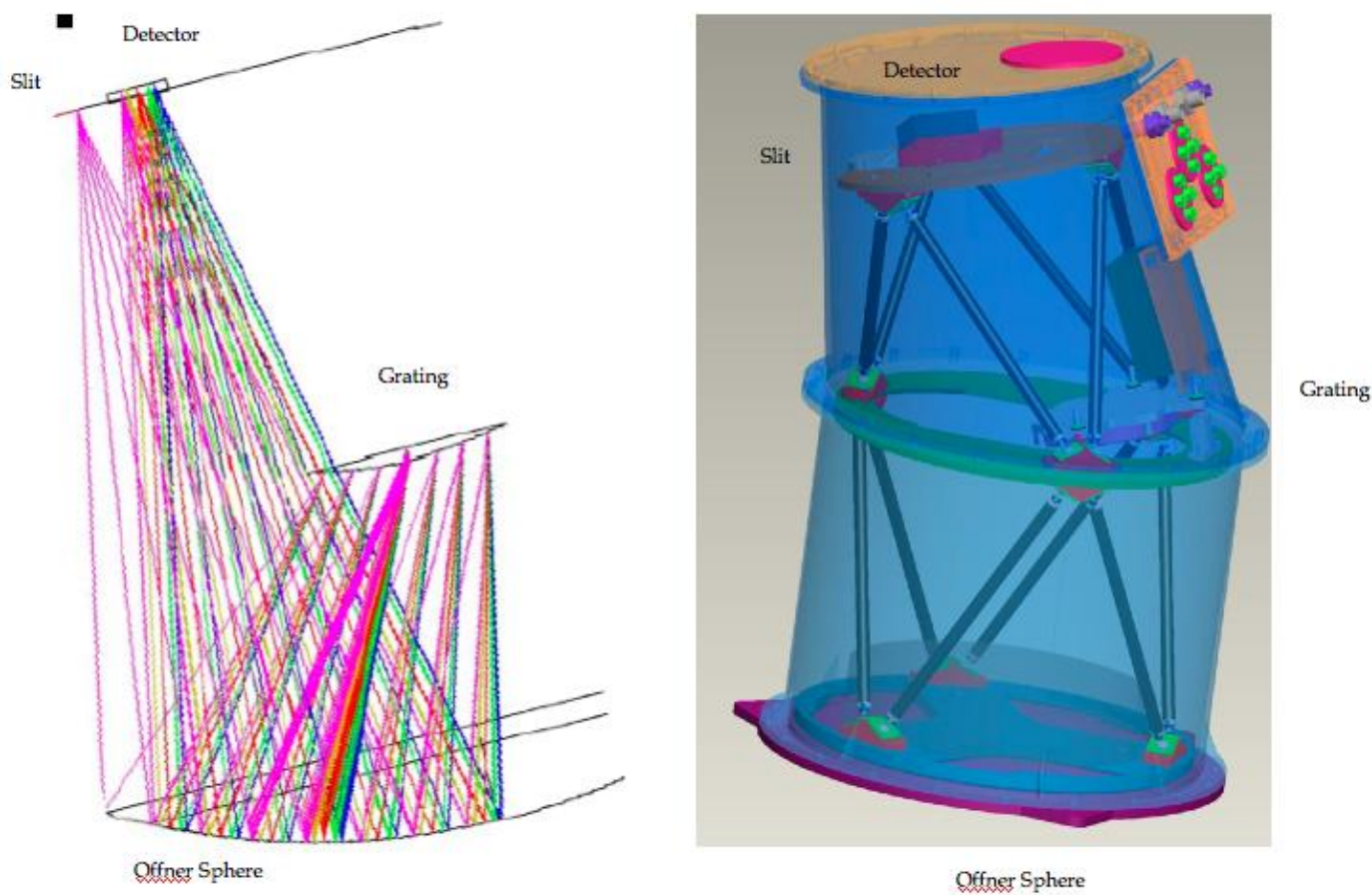

Figure 3: The spectrograph ray trace (left) shows the light entering the system on the upper left (marked slit - furthest pink rays) and returning to the detector, mounted side by side. The assembly drawing on the right shows the entire spectrograph, including the housing. The fibers enter the housing through feedthrough located directly above the slit (roughly where the word "Detector" appears). 


\subsection{Optics}

The spectrograph includes two reflective optics (the Offner sphere and the grating) as well as the fiber bundle (see next section) and the detector. Both the sphere and the grating were mounted using Dow RTV 3145 to secure the optic onto a plate (aluminum for the grating and invar for the sphere). Invar, a nickel steel alloy that has a very low coefficient of thermal expansion, was chosen for the sphere mounting to guarantee no thermal distortion during flight. It was machined to control flexure and to decrease mass. The invar plate was also stabilized with heat treatment during machining.

Two Offner blanks were manufactured by Winlight Optics in Marseille. The first sphere was coated by Tofico with bare aluminum. It had $82 \%$ efficiency at $200 \mathrm{~nm}$ and was used for prototype construction and testing. The second sphere was coated by Goddard Space Flight Center. The coating is optimized for the waveband and is $62 \mathrm{~nm}$ Al overcoated with $41 \mathrm{~nm} \mathrm{MgF} 2$. It was measured to have a reflectance of $92 \%$ at $200 \mathrm{~nm}$. This coating also served as a test run for the large optics (primary and siderostat) used in the FIREBall telescope.

Table 2. Optics Specifications

\begin{tabular}{|r|c|}
\hline \multicolumn{1}{|c|}{ Parameter } & Value \\
\hline Offner Sphere & \\
\hline Diameter & $480 \mathrm{~mm}$ \\
\hline Radius of Curvature & $880 \mathrm{~mm}$ \\
\hline Coating(A1 + MgF2) & $62 \mathrm{~nm}+41 \mathrm{~nm}$ \\
\hline Efficiency (At 200 nm) & $91 \%$ \\
\hline Convex Grating Diameter & $232 \mathrm{~mm}$ \\
\hline Radius of Curvature & $470 \mathrm{~mm}$ \\
\hline Measured efficiency & $20 \%$ \\
\hline
\end{tabular}

The grating blank was also figured by Winlight Optics. The convex grating was holographically ruled by Horiba JobinYvon. The convex shape reduces the spherical aberration introduced by the Offner sphere (fig. 3). A laminar groove profile was used to enhance efficiency. Measured efficiency was found to be lower than that expected from theoretical values. This situation bears some resemblance to an occurrence during the manufacture of the COS gratings for HST $^{5}$. Some combination of groove spacing and the coating appears to be causing a resonance efficiency anomaly ${ }^{6}$. A similar problem seems to have affected the FIREBall grating. This problem is expected to be resolved for the next flight.

The detector is a near-UV flight spare that was built for the GALEX satellite ${ }^{7}$. The microchannel plate (MCP) detector is a photon-counting detector with a wide field of view $(65-70 \mathrm{~mm}$ active diameter) and a quantum efficiency of $6 \%$. The detector operates at high voltage $(-5200 \mathrm{~V})$ and within a sealed vacuum tube. The detector design is optimized for the NUV waveband (1650-3000angstroms) with a fused silica window that has been deposited with a proximity-focused semitransparent $\mathrm{Cs}_{2} \mathrm{Te}$ photocathode ${ }^{8}$. Orthogonal cross delay lines provide incident photon position information from the location of the resulting electron cloud. The detector background is low and is dominated during flight by the sky background $\left(\sim 100\right.$ counts s $\left.^{-1}\right)$ by two orders of magnitude ${ }^{9}$. The detector does not require cooling, allowing for a close fit in the spectrograph housing. 


\subsection{Mount and housing}

The spectrograph is designed using two levels, each with separate carbon hexapod mounts. The lower level holds the grating mount parallel to the bottom of the housing. The upper level allows for correct positioning of the detector and slit with respect to the sphere (figure 3). Each leg was designed to be of adjustable length and to lock. Initial alignment was done using a Faro Arm (50 $\mathrm{m}$ precision) for precise placement matched to measurements from CAD assembly models. Carbon was used for the struts for its strength, relatively low weight, and limited off gassing under vacuum pressures.

The decision was made to enclose the spectrograph in an aluminum pressure vessel. The housing was $1 \mathrm{~m}$ tall with a footprint $0.7 \mathrm{~m}$ in diameter. This housing was designed to keep the pressure and temperature constant as well as to protect the optics from any damage either in flight or upon landing. It added a small amount of weight, but the dominant mass for the spectrograph was the Offner sphere and its internal mount plate. Feedthroughs for the electronics were built into the front panel. The fiber optic bundle entered through the top of the housing (see figure 4). The design allowed for access once the housing was in place through the top cover that had a small port. The majority of adjustments had to be made with the housing removed.

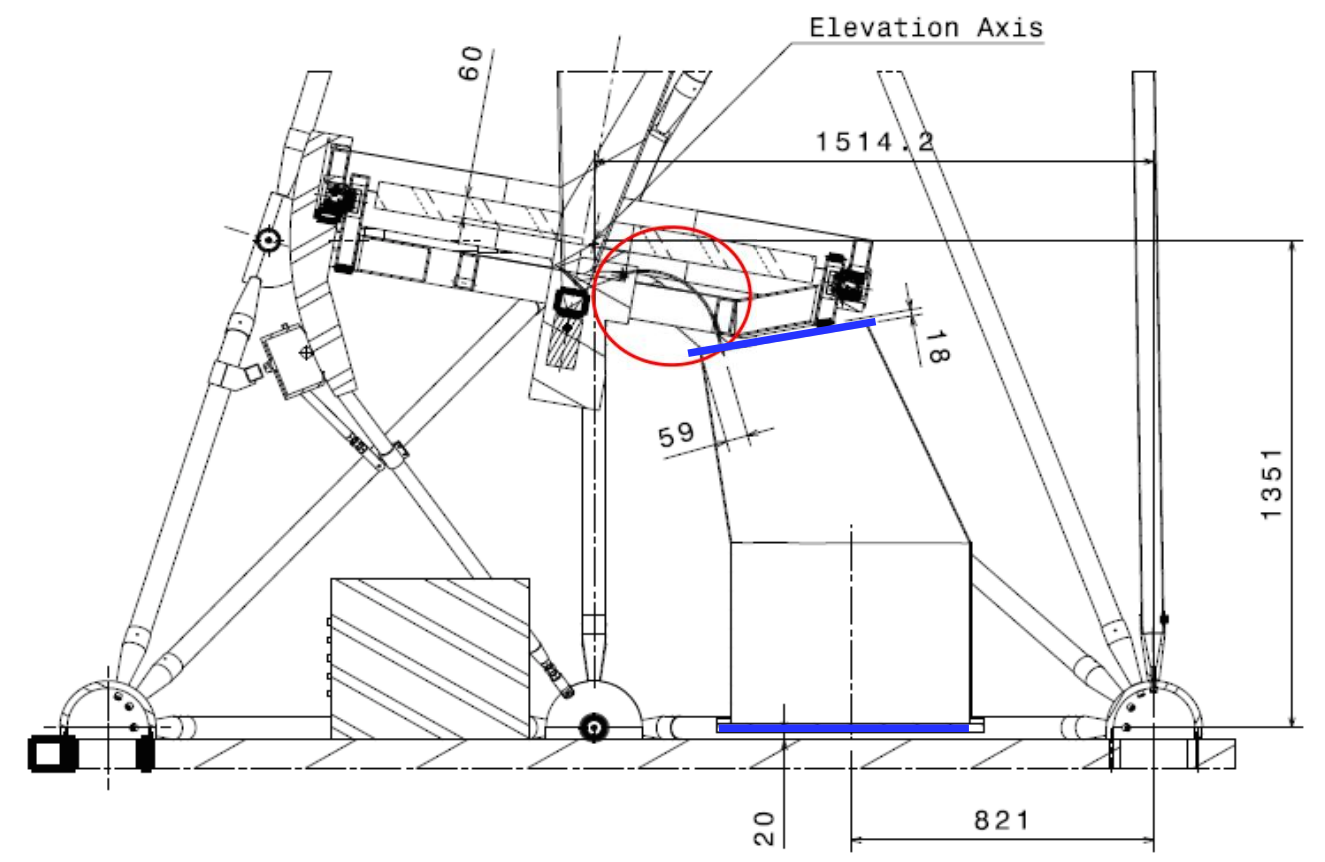

Figure 4: The spectrograph position on the gondola, with respect to the siderostat frame (above it). The top and bottom of the spectrograph can are marked in blue. The fiber bundle can be seen arching to the left out of the top of the spectrograph can (circled in red).

\subsection{Fiber bundle}

Recent advances in fiber manufacturing have decreased absorption caused by solarization and UV defects resulting in increased throughput at the blue and ultraviolet end of the spectral regime $e^{10,11,12}$. The fiber bundle couples the focal plane of the telescope to the entrance slit of the spectrograph, passing through a vacuum feedthrough on the spectrograph housing. The bundle consisted of 342 FVP100110125 fused silica fiber (100 $\mu \mathrm{m}$ core) supplied by Polymicro Technologies. The bundle was tightly packed to a circular aperture at the focal plane end while the spectrograph end was assembled into a slit using subunits mounted individually. Each "tooth" consisted of twenty $58 \mathrm{~cm}$ long science fibers and one $2 \mathrm{~m}$ calibration fiber. The slit was $\sim 50 \mathrm{~mm}$ total length with an optimal radius of curvature of $1200 \mathrm{~mm}$. For this flight the slit was approximately straight. The calibration fibers fed a lamp box mounted on the gondola which 
provided continuum and line emission (Deuterium and PtNe bulbs, respectively). All bundle assembly was done at Columbia University.

The teeth at the slit end of the spectrograph were manufactured first. This gave the opportunity to select units for flatness and surface quality after they were polished, and allowed us to check for broken fibers prior to assembly into the final bundle. Individual fibers were cut to length, with several inches of excess to take into account length lost during bundling and polishing. The slit end was first epoxied using a Delrin mold to fashion a "brick" of fibers. This was then hand polished using decreasing grit size lapping film (from $12 \mu \mathrm{m}$ to $1 \mu \mathrm{m}$ ) and mounted on the aluminum tooth that fit into the slit assembly (see figure 5). Epotek 302-3M was used both for the "brick" and for mounting to the tooth. Each tooth was held by two screws (front and bottom) that allowed the tooth to be adjusted for alignment and focus. The fibers were epoxied to protrude $<1 \mathrm{~mm}$ from the tooth to allow room for touch up polishing without encountering the aluminum surface.
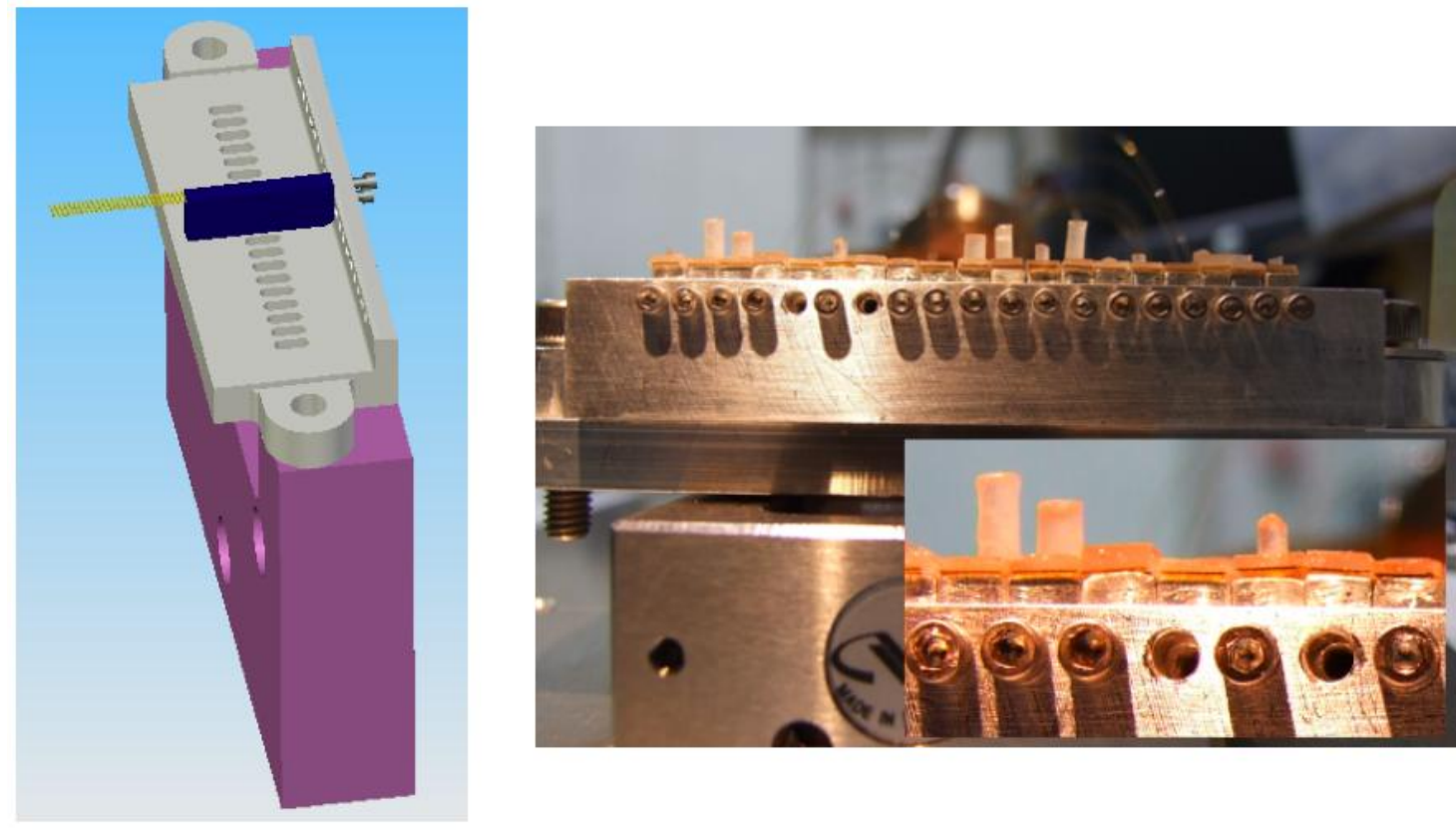

Figure 5: Left - Concept drawing of a single mounted fiber tooth. Right - The fiber slit and a zoom showing deviations from vertical alignment in the slit.

The fibers were next placed through the vacuum feedthrough. After several tests, it was decided to guide fibers through the feedthrough using a thin aluminum mask. Forty fibers (two teeth) were fed through each hole. This helped control the epoxies used to seal the feedthrough while also setting fiber location. The feedthrough was sealed using a two-part process. The first epoxy (AngströmBond AB9001MT) was high viscosity that immobilized the fibers and created an epoxy floor. The second epoxy (AngströmBond AB9110LV) was much lower in viscosity. This allowed the epoxy to flow and fill in any remaining gaps. The second epoxy was much more prone to wicking up the fibers - by applying it second this wicking was almost entirely halted. Wicking decreased flexibility of the final bundle, which would have made final mounting in the spectrograph more difficult. Each application of epoxy was followed by placement in a bell jar. The entire bundle was put under vacuum until visual inspection indicated a cessation of air pocket evacuation (5-7 minutes).

The focal plane end of the bundle was polished last. This was done to protect it from damage and to ensure the largest number of fibers survived assembly. The bundle was hand polished with a specially machined polishing puck. The polishing protocol used was similar to the slit end. A larger grit $(45 \mu \mathrm{m})$ was used initially to clean up fibers that had been trimmed to the correct length after epoxy was applied and cured. All fibers in the terminal end were science fibers (no buffer fibers were used). The interface with the focal plane (see figure 6) was designed to provide the circular aperture and a secure termination point for jacketing materials. 
Two layers of tubing protected the portion of the bundle that was outside of the vacuum vessel. The first was teflon tubing that constricted the diameter of the bundle and also limited sharp bends. The outer layer was a larger stainless steel wrap. This layer protected the bundle from any unintentional contact during integration. The focal plan end terminated in a conical fixture (figure 6) designed to interface repeatably with the focal plane assembly (a dichroic was used to divert optical light to the guider and the fiber bundle sat below it to receive the UV output). The outer tubing was epoxied in place.
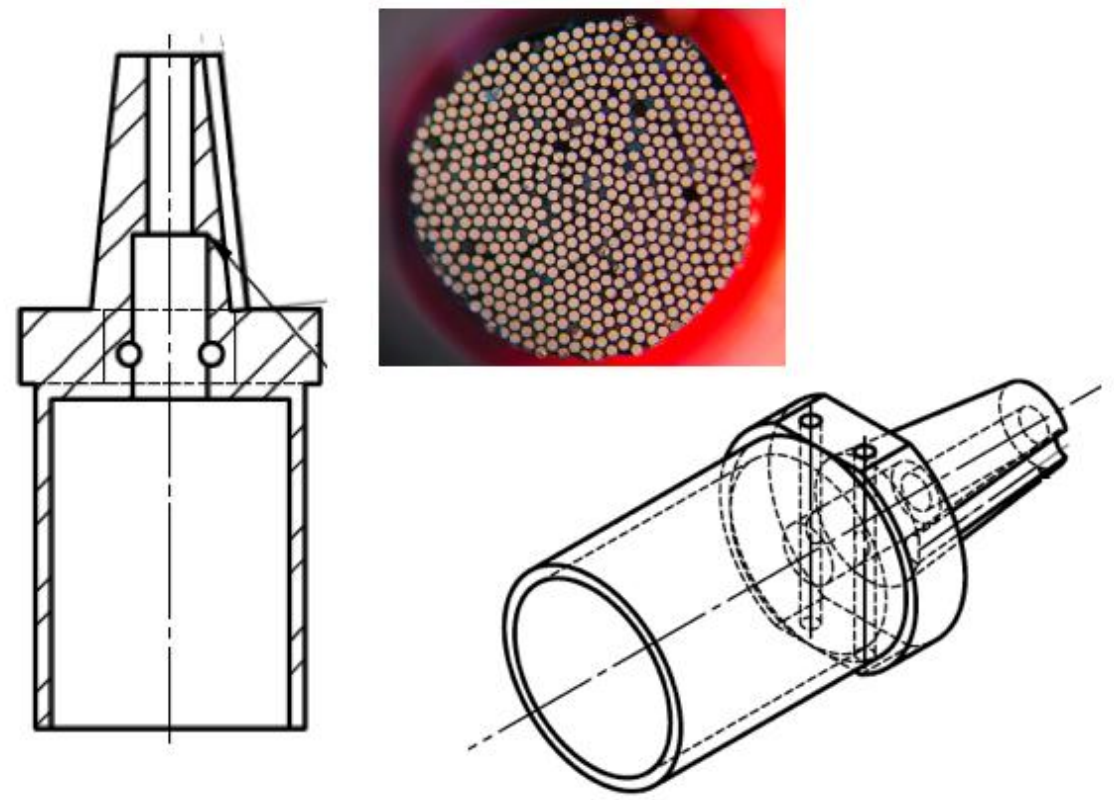

Figure 6: A drawing of the fiber ferrule (left and right bottom) shows the narrowing widths that helped constrict the bundle diameter. The several steps also allowed insertion of Teflon and steel jacketing (one at each step). The picture in the upper right is a test bundle of approximately the same diameter as the flight bundle.

\section{FIRST FLIGHT DETAILS}

\subsection{First flight}

The first flight of FIREBall took place on July $22^{\text {nd }}, 2007$ from the Columbia Scientific Balloon Facility (CSBF, formerly known as NSBF) located in Palestine, TX. The timing of the launch, late in the flight window (a timeline set by the wind patterns and wind speed at float altitude), led to an estimated observational window of approximately 3 hours. Flight time was 6 hours. This included over two hours to reach float altitude. Once the balloon had launched, it was realized that pointing control was not available for the payload. This was later found to be due to a pivot failure. Data was taken for the duration of the flight. The guidance system was able to stabilize for up to two minutes on an acquired target. Spectra were taken of five UV emitting B stars for as long as the tracking would allow (up to one minute) (figure 7). Data was also analyzed to study the ultraviolet sky background. In-flight calibration data is being compared to ground data (figure 8). The current state of knowledge on the UV background comes from past rocket and satellite studies. This work will give insight for others hoping to exploit this gap in the UV emission of the atmosphere for astronomical purposes. 


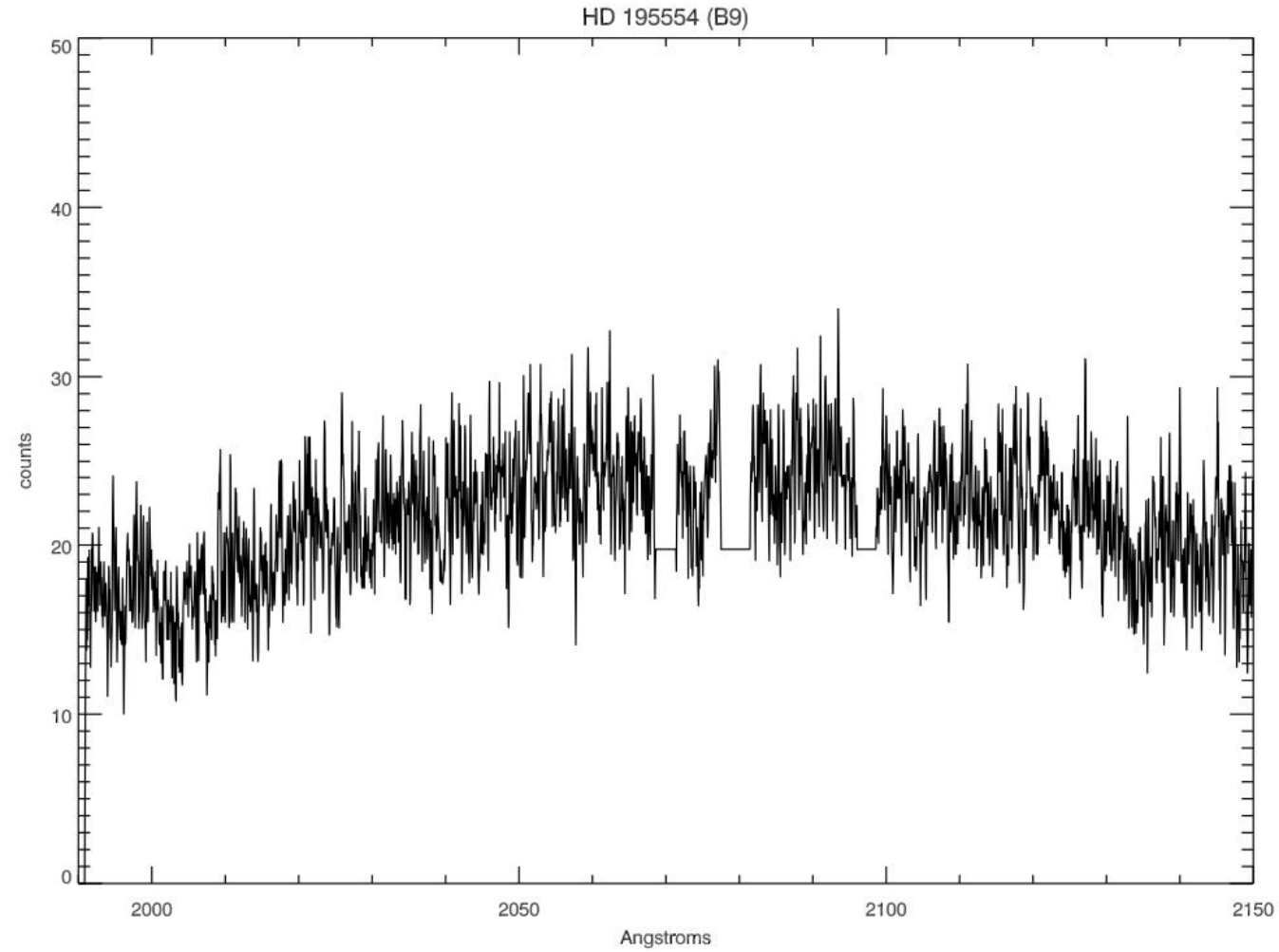

Figure 7: Ultraviolet continuum data from HD 195554, a B9 star observed for 20 seconds. The three flat sections of the spectrum are locations of detector features that have been masked. Total counts $=64,829$

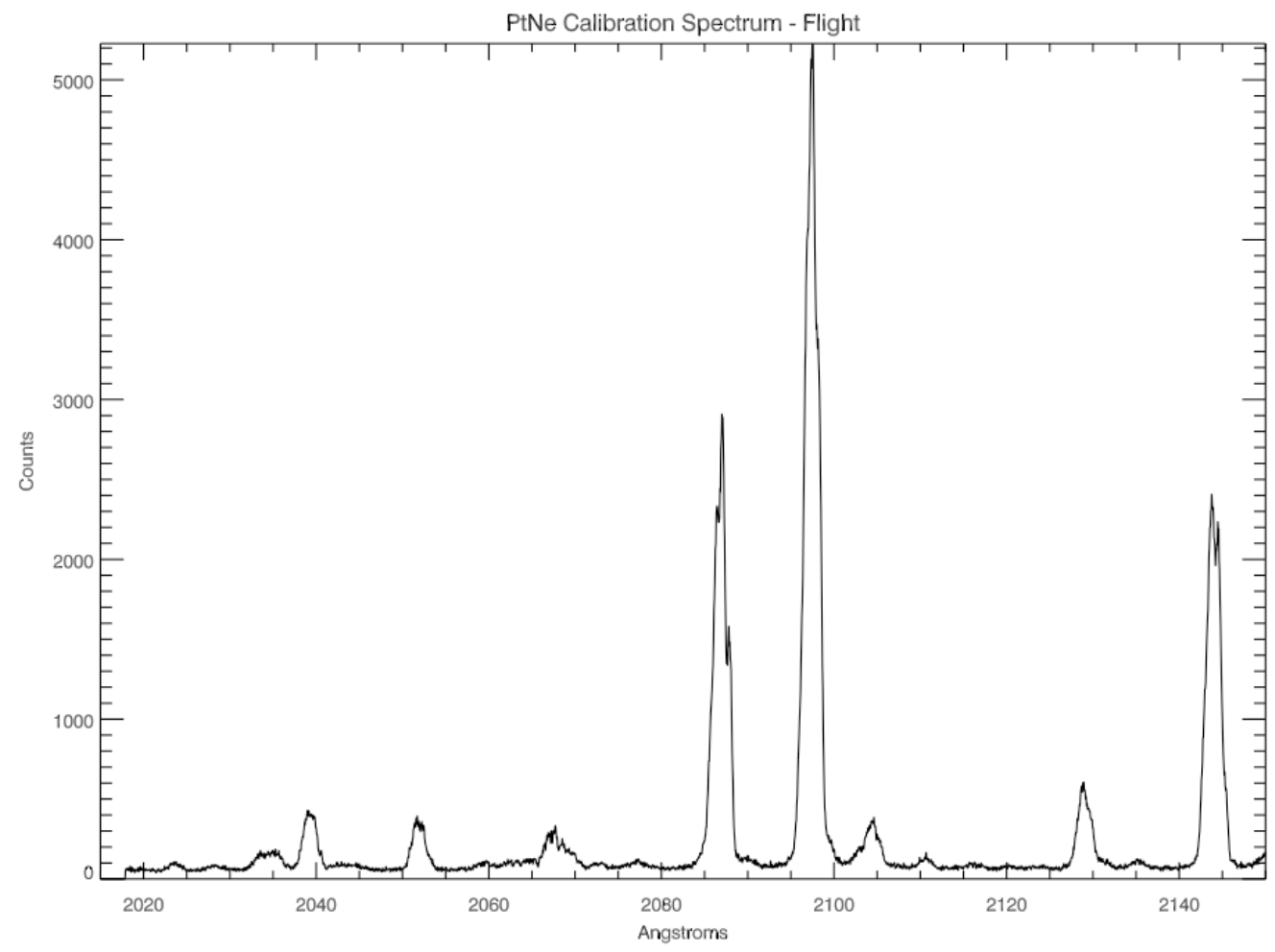

Figure 8: Calibration spectrum of Platinum Neon from the first flight. 
The spectrograph performed as expected under flight conditions. All bonded optics remained secure (both the offner sphere and the grating). The fiber bundle was not damaged. The one failure was a single hexrod leg - a detachment at the ball joint. It was found that this was due to a failure in assembly (misuse of an unwelded ball) rather than any fundamental design flaw.

\section{FUTURE PLANS}

FIREBall is scheduled for at least two more flights in the spring of 2009 and 2010. The 2009 flight will be from Fort Sumner, New Mexico. This launch site gives the possibility of up to 10 hours dark time and 24 hours total flight time. Thermal redesign is required to account for the daylight flight exposure and increased temperature variation. The 2010 flight will take advantage of a planned CSBF Alice Springs, Australia campaign. This campaign contains the possibility of extended dark time as well as compelling targets in the southern sky.

The spectrograph will be upgraded prior to each flight. For the upcoming 2009 flight the following modifications are planned:

- Fiber bundle manufacturing and calibration overhaul (including review of bundle strain ${ }^{13}$ )

- New Grating (Change in groove density and coating)

- Internal mount upgrade for improved hexleg locking and precision

- Thermal study \& possible redesign

- Baffling/Blacking to further reduce scattered light

\section{CONCLUSION}

The first flight of FIREBall was a successful engineering test of a balloon borne fiber-fed UV spectrograph, demonstrating the soundness of the concept and the system. FIREBall has two future flights scheduled in the spring of 2009 and again in 2010. There are also plans for a second generation of FIREBall, incorporating upgrades such as a higher efficiency detector. Balloon flights continue to be an excellent proving ground for novel UV astronomical instrumentation.

\section{ACKNOWLEDGEMENTS}

The authors would like to thank Victoria Johnston, David Stenning, and Eve LoCastro for their hard work in the lab. The crew at CSBF was fantastic and make launching a balloon seem (almost) easy.

\section{REFERENCES}

[1] Davé, R., Cen, R., Ostriker, J.P., Bryan, G.L., Hernquist, L., Katz, N., Weinberg, D.H., Norman, M.L., and O’Shea, B., "Baryons in the Warm-Hot Intergalactic Medium", ApJ, 552(2), 473-483 (2001).

[2] Huffman, R. E., LeBlanc, F.J., Larrabee, J.C., and Paulsen, D.E., "Satellite Vacuum Ultraviolet Airglow and Auroral Observations" JGR, 85(A5), 2201-2215 (1980).

[3] Furlanetto, S. R., Schaye, J., Springel, V., and Hernquist, L., "Ultraviolet Line Emission From Metals In The Low Redshift Intergalactic Medium" ApJ, 606, 221-236 (2004).

[4] Grange, R., Milliard, B., McLean, R., Martin, C., Deharveng, J.M., and Schiminovich, D., "Three-dimensional spectroscopy with a fiber-fed NUV spectrograph”, Proc. SPIE 5898, (2005)

[5] Kuznetsov, I. G., Wilkinson, E., Content, D.A., Boucarut, R.A., and Madison, T.J., "Grating efficiences comparison study: calculations versus metrology for various types of high groove density gratings at VUV-UV wavelengths", Proc. SPIE 5178, 267-277 (2004).

[6] Goray, L. I., Kuznetsov, I.G., Sadov, S.Y., and Content, D.A., "Multilayer resonant subwavelength gratings: effects of waveguide modes and real groove profiles", J.Opt. Soc. Am. A 23(1), 155-164 (2006).

[7] Morrissey, P., Conrow, T., Barlow, T.A., Small, T., Seibert, M., Wyder, T.K., Budavari, T., Arnouts, S., Friedman, 
P.G., Forster, K., Martin, D.C., Neff, S.G., Schiminovich, D., Bianchi, L., Donas, J., Heckman, T.M., Lee, Y., Madore, B.F., Milliard, B., Rich, R.M., Szalay, A.S., Welsh, B.Y., and Yi, S.K., "The Calibration and Data Products of GALEX", ApJSS 173, 682-697 (2007).

[8] Siegmund, O., Welsh, B., Martin, C., Barlow, T., Bianchi, L., Byun, Y., Forster, K., Friedman, P.G., Jelinsky, P., Madore, B., Malina, R., Milliard, B., Morrissey, P., Neff, S., Rich, M., Schiminovich, D., Small, T., Szalay, A., and Wyder, T., "The GALEX mission and detectors", Proc. SPIE 5488, 13-24 (2004).

[9] Morrissey, P., Schiminovich, D., Barlow, T.A., Martin, D.C., Bakkolb, B., Conrow, T., Cooke, B., Erickson, K., Fanson, J., Friedman, P.G., Grange, R., Jelinsky, P.N., Lee, S., Liu, D., Mazer, A., McLean, R., Milliard, B., Randall, D., Schmitigal, W., Sen, A., Siegmund, O.H.W., Surber, F., Vaughan, A., Viton, M., Welsh, B.Y., Bianchi, L, Byun, Y., Donas, J., Forster, K., Heckman, T.M., Lee, Y., Madore, B.F., Malina, R.F., Neff, S.G., Rich, R.M., Small, T., Szalay, A.S., and Wyder, T.K., "The On-Orbit Performance Of The Galaxy Evolution Explorer", ApJ 619, L7-L10 (2005)

[10] Klein, K. F., Arndt, R., Hillrichs, G., Ruetting, M., Veidemanis, M., Dreiskemper, R., Clarkin, J., and Nelson, G., "UV Fibers For Applications Below 200nm", Proc. SPIE 4253, (2001).

[11] Wang, T., Guo, X., and Chen, Z., "Improved silica-core fibers in the UV-transmission", Proc. SPIE 5623, (2005).

[12] Khalilov, V. K., Klein, K., Belmahdi, J., Timmerman, R., and Nelson, G., "High-OH fibers with higher stability in the UV-region", Proc. SPIE 6083, (2006).

[13] Kelz, A., Verheijen, M., Roth, M.M., Laux, U., and Bauer, S. (2004). "Development of the wide-field IFU PPak", Proc. SPIE 5492, 719-730(2004) 